\title{
Erratum: Nonequilibrium Phase Behavior from Minimization of Free Power Dissipation [Phys. Rev. Lett. 117, 208003 (2016)]
}

\author{
Philip Krinninger, Matthias Schmidt, and Joseph M. Brader \\ (Received 5 June 2017; published 13 July 2017)
}

DOI: 10.1103/PhysRevLett.119.029902

In deriving the nonequilibrium phase coexistence conditions (8) via the double tangent construction (7), as exemplified in Fig. 2(b) and leading to the results in Fig. 3, we assumed the concept of minimization of free power dissipation, $R_{t}[\rho, \mathbf{J}]$. However, while minimization of $R_{t}[\rho, \mathbf{J}]$ with respect to the current distribution $\mathbf{J}(\mathbf{r}, \boldsymbol{\omega}, t)$ generates a one-body force balance equation of motion (1), we had implicitly assumed [1] that the functional is also minimal with respect to the density distribution $\rho(\mathbf{r}, \boldsymbol{\omega}, t)$. This additional minimization is not present in power functional theory [8], which rather states that

$$
\frac{\delta R_{t}[\rho, \mathbf{J}]}{\delta \rho(\mathbf{r}, \boldsymbol{\omega}, t)}=\alpha(\mathbf{r}, \boldsymbol{\omega}, t)
$$

where $\alpha(\mathbf{r}, \boldsymbol{\omega}, t)$ is a Lagrange multiplier corresponding to the constraint between $\rho(\mathbf{r}, \boldsymbol{\omega}, t)$ and $\mathbf{J}(\mathbf{r}, \boldsymbol{\omega}, t)$ that is imposed by the continuity equation.

For our test case of motility-induced phase separation in active Browian particles, recent simulation data [2,3] very clearly points to the fact that both $\pi$ and $\nu$ possess different values in the coexisting phases. Hence the conclusion that (8) offers a shortcut to phase coexistence, bypassing the need to solve the force balance equation (1) across the interface, is not valid. This does not affect the validity of the excess dissipation functional (14), as demonstrated in Figs. 1 and 2, which hence offers a practical way to theoretically address the interfacial problem.

We thank D. de las Heras, T. Speck, J. Rodenburg, R. van Roij, S. Paliwal, and M. Dijkstra for useful discussions.

[1] J. Rodenburg and R. van Roij (private communication).

[2] S. Paliwal and M. Dijkstra (private communication).

[3] T. Speck (private communication). 